\title{
Spin Labels in the Investigation of Macromolecules Adsorption on Magnetic Nanoparticles
}

\author{
A.V. Bychkova ${ }^{1}$, O.N. Sorokina ${ }^{1}$, A.B. Shapiro ${ }^{1}$, A.P. Tikhonov ${ }^{2}$ and A.L. Kovarski ${ }^{*}, 1$ \\ ${ }^{1}$ Emanuel Institute of Biochemical Physics RAS, Kosygin str., 4, Moscow, 119334, Russia \\ ${ }^{2}$ Mendeleev University of Chemical Technology of Russia, Miusskaya sq., 9, 125047, Russia
}

\begin{abstract}
The new application aspect of spin labels technique to macromolecular adsorption on magnetic nanoparticles and quantitative adsorption layer characterization was developed. The technique is based on measurement of ESR signal intensity of stable nitroxide radicals, covalently bound to macromolecules. As a result of spin-labeled macromolecules adsorption on the nanoparticles surface ESR spectra lines broaden due to dipole-dipole interaction of unpaired electrons of radical with particles magnetic moment. This leads to signal intensity decreasing. Multilayer adsorption of biological macromolecules - fibrinogen and albumin, and also synthetic polymer - polyethyleneimine on magnetite particles with average diameter $17 \mathrm{~nm}$ was studied. It was shown that the method is applicable for adsorbed macromolecules number evaluation when the thickness of multilayer coat is up to $40 \mathrm{~nm}$.
\end{abstract}

Keywords: fibrinogen, albumin, polyethyleneimine, nanoparticles, magnetite, adsorption, spin labels, ESR spectroscopy.

\section{INTRODUCTION}

Adsorption properties of metal-containing nanoparticles including nanoparticles with magnetic moment are still poorly studied in contrast to traditional organic and nonorganic sorbents. At the same time magnetic nanoparticles are used either as fillers for composite materials or in medicine for delivery of drugs to diseased areas. Adsorption properties of these particles in both cases play an important role. It was shown previously that the usage of paramagnetic molecules (spin labels) as sensors allows one not only to study microparticles adsorption capacity but also gain information about adsorption layer properties [1-5]. The aim of this work was to make an analysis of spin labels technique capability in the study of macromolecules adsorption on magnetic nanoparticles surface. Adsorption of protein molecules (fibrinogen FG and bovine serum albumin BSA) and synthetic polymer polyethyleneimine PEI on magnetite nanoparticles in water medium have been studied.

\section{MATERIALS AND METHODOLOGY}

\section{Materials}

Magnetite nanoparticles were synthesized by coprecipitation of $\mathrm{Fe}^{2+}$ and $\mathrm{Fe}^{3+}$ salts. The particles were stabilized by the double electric layer in phosphate-citrate buffer according to the procedure [6]. These nanoparticles were characterized by lognormal distribution in diameters. The average value of $d$ was $17 \mathrm{~nm}$, half-height width was 12 $\mathrm{nm}$. Molecular weights of FG and BSA (both Sigma, USA) were 340 and $66 \mathrm{kDa}$, respectively [7]. PEI with molecular weight $10 \mathrm{kDa}$ was produced in Russian Institute of Petrochemical Synthesis. Hydrosol of nanoparticles was mixed with macromolecular solution in water (PEI) or in

*Address correspondence to this author at the Mendeleev University of Chemical Technology of Russia, Miusskaya sq., 9, 125047, Russia;

E-mail: alkogon383@yandex.ru phosphate buffer with $\mathrm{pH} \sim 8.5$ (proteins). Concentration of macromolecules came to $7 \mathrm{mg} / \mathrm{ml}$ for FG, $10 \mathrm{mg} / \mathrm{ml}$ for BSA and $0.15 \mathrm{mg} / \mathrm{ml}$ for PEI. The solutions were exposed at room temperature for $1 \mathrm{~h}$. Nanoparticles concentrations in soles used were 0.005 and 0.01 vol.\%.

\section{Spin Labels Binding Procedure}

The spin labels are stable nitroxide radicals covalently bonded to macromolecules. The information about spin labels concentration and rotational correlation time $\tau$ which characterizes the intensity of molecular motion was obtained from electron spin resonance (ESR) spectra [8]. Stable chlorinecontaining nitroxide radical $\left(\mathrm{R}_{\mathrm{I}}\right)$ was used as spin label:

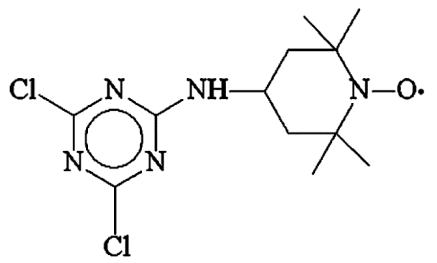

Spin-labeled proteins were obtained by $\mathrm{R}_{\mathrm{I}}$ binding to amino groups of proteins (BSA and FG) in phosphate buffer with $\mathrm{pH} \sim 8.5$ according to the reaction [9]:<smiles>CCCONCON1C(C)(C)CC(Nc2nc(Cl)nc(Cl)n2)CC1(C)C</smiles><smiles>CON1C(C)(C)CC(Nc2nc(Cl)nc(NNCC(Cl)Cl)n2)CC1(C)C</smiles> 
The reaction was conducted at room temperature. The radical (molecular weight is $319 \mathrm{~g} / \mathrm{mol}$ ) in amount of $0.9 \mathrm{mg}$ or $1.8 \mathrm{mg}$ was dissolved in $1 \mathrm{ml}$ of $96 \%$ ethanol. $25 \mu \mathrm{l}$ of label solution was added to $1 \mathrm{ml}$ of protein solution in phosphate buffer with $\mathrm{pH} \sim 8.5$. Label-containing solution was incubated for two hours. Then, it was dialyzed on magnetic stirrer for 12 hours with changing of buffer solution for three times. As a result of the reaction spin-labeled proteins were obtained. The average number of labels per molecule were 3 for $\mathrm{FG}$ and 1 for BSA.

Spin-labeled PEI was also obtained by $\mathrm{R}_{\mathrm{I}}$ binding to amino groups of the polymer in water solution at room temperature. The radical in amount of $1.5 \mathrm{mg}$ was dissolved in $96 \%$ ethanol $(5 \mathrm{ml}) .25 \mu \mathrm{l}$ of label solution was added to $1 \mathrm{ml}$ of PEI solution in water so that 10 labels could be bonded to one polymer molecule. Label-containing solution was incubated for a day which is sufficient for labels to be bonded.

\section{ESR Spectra Registration and Analysis}

ESR spectra were recorded using Bruker EMX 2.7/8 X-band spectrometer at temperature $293 \pm 0.5 \mathrm{~K}$. Microwave power was $5 \mathrm{~mW}$, modulation amplitude $2 \mathrm{G}$.

ESR spectra of the systems magnetite - spin labeled macromolecules consist of three narrow lines of nitroxide radical and wide line (700-900 G) of magnetite nanoparticles [10]. The example of the spectrum for spin labeled PEI in the presence of magnetite nanoparticles is shown in Fig. (1). ESR spectra of spin labels were obtained by subtraction of magnetite lines from total spectra in a manner described in [10]. The following parameters were defined from the ESR spectra of spin labels: central component intensity $\left(I_{0}\right)$ and linewidth $\left(\Delta H_{0}\right)$, rotational correlation time $(\tau)$. Values of $\tau$ were determined by matching the experimental spectra to the theoretical, calculated in [11]. Magnesium oxide powder containing $\mathrm{Mn}^{2+}$ ions was used as an external standard.

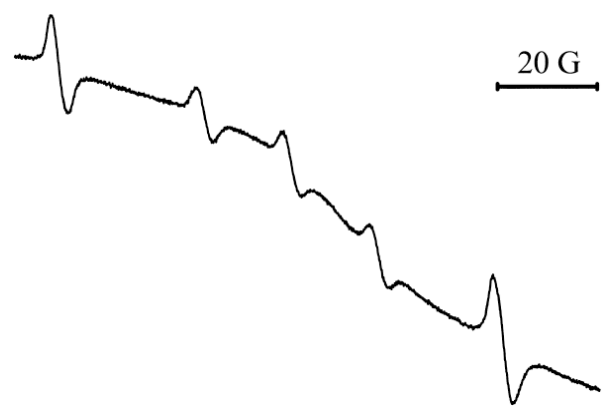

Fig. (1). ESR spectra of spin-labeled PEI in water medium with nanoparticles in concentration 0.005 vol.\%. The outer lines belong to $\mathrm{Mn}^{2+}$ standard. $\mathrm{T}=293 \mathrm{~K}$.

\section{RESULTS AND DISCUSSION}

\section{Spin Label Technique Data}

The spectra of radical $R_{I}$ and spin labels bonded to protein and PEI molecules are shown in Fig. (2).

Correlation times $\tau$ are:

- $\quad 1 \cdot 10^{-10} \mathrm{~s}$ for free radical rotation in solution,
- $\quad 2 \cdot 10^{-7} \mathrm{~s}$ for rotation of spin labels bonded to BSA molecules;

- $\quad 2 \cdot 10^{-9} \mathrm{~s}$ for rotation of spin labels bonded to $\mathrm{FG}$ molecules;

- $\quad 3.3 \cdot 10^{-10} \mathrm{~s}$ for rotation of spin labels bonded to PEI molecules.

Comparison of $\tau$ values presented above allows one to make following conclusions: radical binding to macromolecules restricts its rotational mobility and the difference between label mobility for BSA, FG and PEI exists. The slowest mobility is typical for labels bonded to BSA, the most rapid mobility is the feature for lowmolecular PEI. The intermediate $\tau$ value is peculiar to spin labels on FG. These results can be explained by the diversity in macromolecules structure and molecular weigh. BSA has a globular structure, and, probably, considerable part of labels can be located inside the globule, where local viscosity is high, whereas the labels bonded to fibrous protein (FG) molecules are in the areas with more incoherent packing. Spin labels are predominantly surrounded by water molecules in low-molecular PEI solutions, so that their rotational mobility is restricted mainly by rotational barrier about ordinary chemical bond.

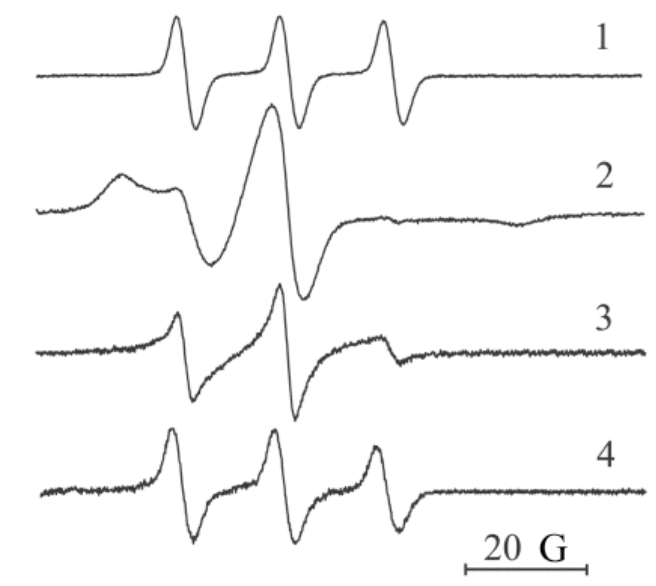

Fig. (2). ESR spectra of solutions of radical $R_{I}(1)$, spin-labeled: BSA (10 mg/ml) (2), FG (7 mg/ml) (3) and PEI (4). T = $293 \mathrm{~K}$.

Dipole-dipole interaction between radical and nanoparticle depends mostly on the distance between these species. The dipole line broadening of radical spectra $\delta H$ can be calculated by equation $[12,13]$ :

$\delta H=\frac{4 \pi}{3 \sqrt{3}} \frac{\left\langle\mu_{z}\right\rangle}{r^{3}}$,

where $r$ is the distance between radical and nanoparticle, $<\mu_{\mathrm{z}}>$ is the average magnetic moment of nanoparticle, equals to $4 \cdot 10^{-16} \mathrm{G} \cdot \mathrm{cm}^{3}$ [14].

The line broadening dependence on the distance between radical and nanoparticle is shown in Fig. (3). According to equation (1) and Fig. (3), the sharp line broadening takes place if paramagnetic and magnetic particles are on the distance less than $40 \mathrm{~nm}$. The line width may grow up to 80 $\mathrm{G}$ that leads to line intensity hundreds times decreasing. 


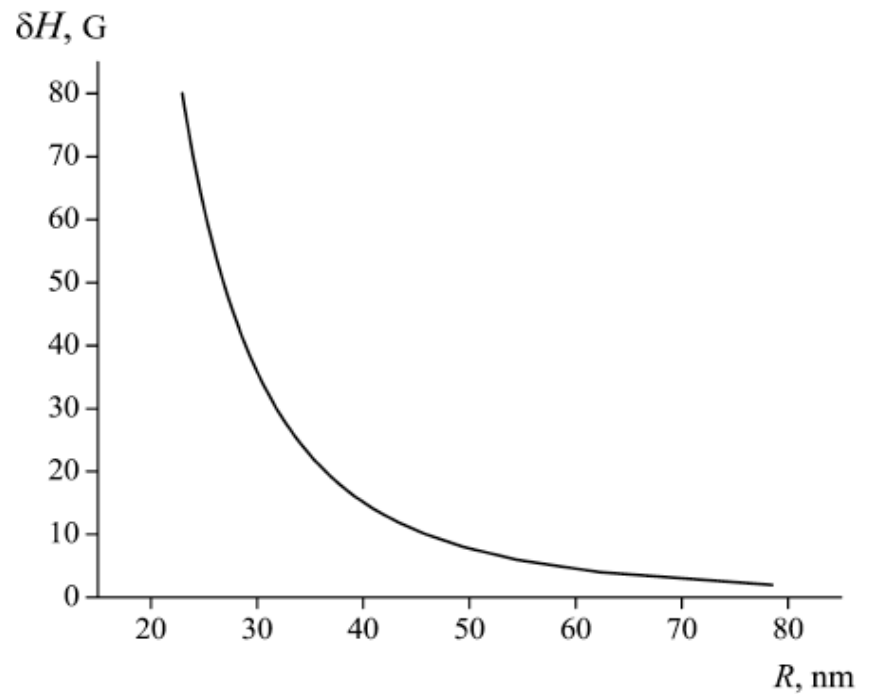

Fig. (3). Line broadening dependence on the distance between radical and nanoparticle.

Under the stipulation of statistical distribution of the radicals in solution at the concentration of particles 0.01 vol. $\%$ there are less than $2 \%$ of radicals in the surface layer of $40 \mathrm{~nm}$ in thickness. It is evident, that considerable changes in ESR linewidth and intensity will not be observed in this case. This fact is proved by the data presented in Table 1. It is important to note that the results obtained do not depend on paramagnetic particles concentration if these particles are distributed in solution statistically.

If the radicals (or labels) adsorb on nanoparticles surface, the amount of radicals in the surface layer grows and the condition of radical uniform distribution breaks. ESR lines of these radicals broaden and, therefore, their intensity decreases sharply. Such lines will not be seen against the background of the line from radicals dispersed in solution. Apparent line intensity will also decrease. So, analyzing line intensity changes we can detect the adsorption of the macromolecules and estimate the low limit of adsorption layer thickness. Denoting $n_{a}$ is the amount of adsorbed radicals and $n$ is the total amount of radicals in solution, we can right down:

$\frac{n_{a}}{n}=\frac{I-I^{\prime}}{I}$

where $I$ and $I^{\text {, }}$ are ESR line intensity before and after nanoparticles introduction into the solution.
For spin labeled macromolecules, signal intensity decreasing after introduction of magnetic nanoparticles into the solution is a result of macromolecule adsorption. The dependences of spectra lines intensity for spin labeled BSA, FG and PEI molecules on nanoparticles concentration in solution are presented in Table $\mathbf{1}$.

It is seen from Table $\mathbf{1}$ that the intensity of ESR signal of free radicals and labels bonded to BSA does not change when nanoparticles concentration in the system changes. However, label signal intensity in the case of FG decreases to $67 \%$ and in the case of PEI to $28 \%$. These results show that FG and PEI are adsorbed and lines of labels bonded to them are abruptly broadened. Equation (2) allows estimating the amount and fraction of adsorbed radicals. The fact that signal intensity of spin-labeled BSA does not change in the presence of nanoparticles means either this protein does not adsorb or the thickness of adsorption layer is insignificant. Since ESR signal intensity is the main quantitative parameter we should estimate the error in $I$ value. The basic contribution to imprecision is predicated upon procedure validity of magnetic nanoparticles and paramagnetic labels spectra division. It follows from spectrum analysis that the error in $I$ value equals to $\sim 6 \%$.

We should note that adsorbed molecules fraction coincide with adsorbed radicals fraction obtained from Eq. (2). The amount of FG molecules adsorbed was calculated from adsorbed radicals fraction (33\%), nanoparticles concentration ( 0.01 vol.\%), FG concentration $(7 \mathrm{mg} / \mathrm{ml})$ and nanoparticles density $\left(5.2 \mathrm{~g} / \mathrm{cm}^{3}\right)$. The results obtained showed that 95 protein molecules adsorbed on one nanoparticle. The diameter of protein coat formed around the nanoparticle was calculated in consideration that FG molecules adsorbed on nanoparticle occupy a volume equaled to production of their amount and one molecule volume (2900 $\mathrm{nm}^{3}$ according to [15]). If molecules formed a close packing on the nanoparticles surface, the diameter of particle along with coat equaled to $91 \mathrm{~nm}$ and layer thickness of $\mathrm{FG}$ coat equaled to $\sim 32 \mathrm{~nm}$. The adsorption layer thickness calculated this way was minimal, because we considered that molecules on nanoparticles surface had close packing.

PEI molecules number in adsorption layer was calculated the same way. It was shown that there were approximately 167 PEI molecules per nanoparticle at concentration 0.01 vol.\%. PEI concentration was $0.15 \mathrm{mg} / \mathrm{ml}$. Adsorption layer thickness calculated in consideration of close packing of

Table 1. ESR Spectra Central Line Linewidth $\Delta H_{0}$ and Intensity $I_{0}$ of Radical $R_{I}$ and Spin Labels on Macromolecules Depending on Nanoparticles Concentration in Solution

\begin{tabular}{|c|c|c|c|c|c|c|c|c|}
\hline \multirow{2}{*}{$\begin{array}{l}\text { Nanoparticles } \\
\text { Concentration } \\
\text { in Solution, Vol. \% }\end{array}$} & \multicolumn{2}{|c|}{$\mathbf{R}_{\mathbf{I}}^{*}$} & \multicolumn{2}{|c|}{ Labels on BSA** } & \multicolumn{2}{|c|}{ Labels on FG ** } & \multicolumn{2}{|c|}{ Labels on PEI *** } \\
\hline & $\Delta H_{0} \pm 0.3, G$ & $\begin{array}{c}I_{0} \pm 0.03 \\
\text { Relative } \\
\text { Units }\end{array}$ & $\Delta H_{0} \pm 0.3, G$ & $\begin{array}{c}I_{0} \pm 0.03 \\
\text { Relative } \\
\text { Units }\end{array}$ & $\Delta H_{0} \pm 0.3, G$ & $\begin{array}{c}I_{0} \pm 0.03 \\
\text { Relative } \\
\text { Units }\end{array}$ & $\Delta H_{0} \pm 0.3, G$ & $\begin{array}{c}I_{0} \pm 0.03 \\
\text { Relative } \\
\text { Units }\end{array}$ \\
\hline 0.000 & 3.2 & 1.00 & 5.9 & 1.00 & 5.5 & 1.00 & 3.4 & 1.00 \\
\hline 0.005 & 3.5 & 0.95 & 5.6 & 1.03 & 5.2 & 0.88 & 3.5 & 0.44 \\
\hline 0.010 & 3.2 & 1.00 & 5.8 & 0.97 & 5.3 & 0.67 & 3.2 & 0.28 \\
\hline
\end{tabular}

$* c=8 \cdot 10^{16} \mathrm{spin} / \mathrm{ml}$.

** In water buffer, pH 8.5

$* * *$ In water medium. 
molecules (according to [16] molecule volume is $10 \mathrm{~nm}^{3}$ ) equaled to $1.5 \mathrm{~nm}$.

\section{Estimation of Dipole-Dipole Contribution to Line Broadening Due to Interaction Between Spin Labels}

We should note that when concentration of spin labels on immobilized macromolecules is high the dipole interaction between radicals also leads line broadening. Dipole broadening can be calculated by equation [17-19]:

$$
\delta H_{d d}=c_{r} k_{d d},
$$

where $c_{r}$ is a concentration of spin labels, $k_{d d}$ is a constant of dipole broadening equals to $3.5 \cdot 10^{-20} \mathrm{G} \cdot \mathrm{cm}^{3}$. In the case of PEI the distance between labels is as small as $\sim 1 \mathrm{~nm}$ and the value of dipole broadening amounts to $35 \mathrm{G}$ (if spinexchange narrowing is ignored). When adsorption on particles with vast magnetic moment takes place the lines broaden more significantly. So, despite of the type of broadening mechanism the lines of spin-labeled macromolecules immobilized on surface will not appear in the spectrum. Interaction between radicals should be taken into account only when adsorption on nonmagnetic particles is studied. If the concentration of spin labels is low dipole interaction between them is so weak that it will not lead to line broadening. For example, in the case of FG such broadening equals to $0.035 \mathrm{G}$.

\section{Linear Aggregates Formation in the System Proteins- Nanoparticles in External Magnetic Field}

It is known that under the influence of direct magnetic field linear nanoparticles chains orientated along the flux lines are formed in magnetic particles dispersions $[6,20,21]$. The number and length of these chains are defined by nanoparticles concentration and magnetic moment, their size and shape, magnetic field strength and temperature. In the present work it was shown that large linear aggregates consisting of both nanoparticles and protein molecules connected with them are formed. Photos of these linear aggregates in three different systems are presented in Fig. (4).
The large linear structures formed in the flat cell containing FG $(3 \mathrm{mg} / \mathrm{ml})$ solution are shown in Fig. (4b). The magnetite nanoparticles concentration was 0.01 vol.\%, magnetic field strength was $1.5 \mathrm{kG}$ and exposure time of 1 hour. Under the same conditions and exposure time of 2.5 hours aggregates of significantly less size formed in BSA $(10 \mathrm{mg} / \mathrm{ml})$ solution (Fig. 4c). In the absence of protein linear aggregates were also registered after 2.5 hour exposure (Fig. 4a). Therefore, magnetic nanoparticles contribute to self-assembly of protein molecules in magnetic field. This self-assembly takes place both in longitudinal and normal direction so that large anisotropic structures form. The formation of longest chains in the case of FG may be explained by strong interaction of side domains of FG fibrils adsorbed on neighboring nanoparticles. After switching off the magnetic field these structures transform to large spheroids.

In the system containing PEI-coated nanoparticles the linear structures were not registered.

\section{CONCLUSION}

The data obtained in this study showed the efficiency of spin labels technique in research of macromolecules adsorption on metal-containing nanoparticles. The adsorption of spin-labeled macromolecules on magnetic nanoparticles leads to decreasing of ESR signal intensity. This approach allows defining the fraction of adsorbed macromolecules and estimating multilayer coat thickness, if it does not exceed $40 \mathrm{~nm}$. The error in adsorbed molecules fraction determination is less than $6 \%$. It ought to be noted that the procedure of nitroxide radical binding to reactive groups of macromolecules is not difficult and not timeconsuming. As it mentioned in experimental section, radical $\mathrm{R}_{\mathrm{I}}$ binding to amino-groups is carried out at room temperature during 1 day.

The results concerning FG adsorption on magnetite nanoparticles obtained in this work are supported by dynamic light scattering data. Thus, according to light scattering, 95-100 FG molecules adsorb per nanoparticle

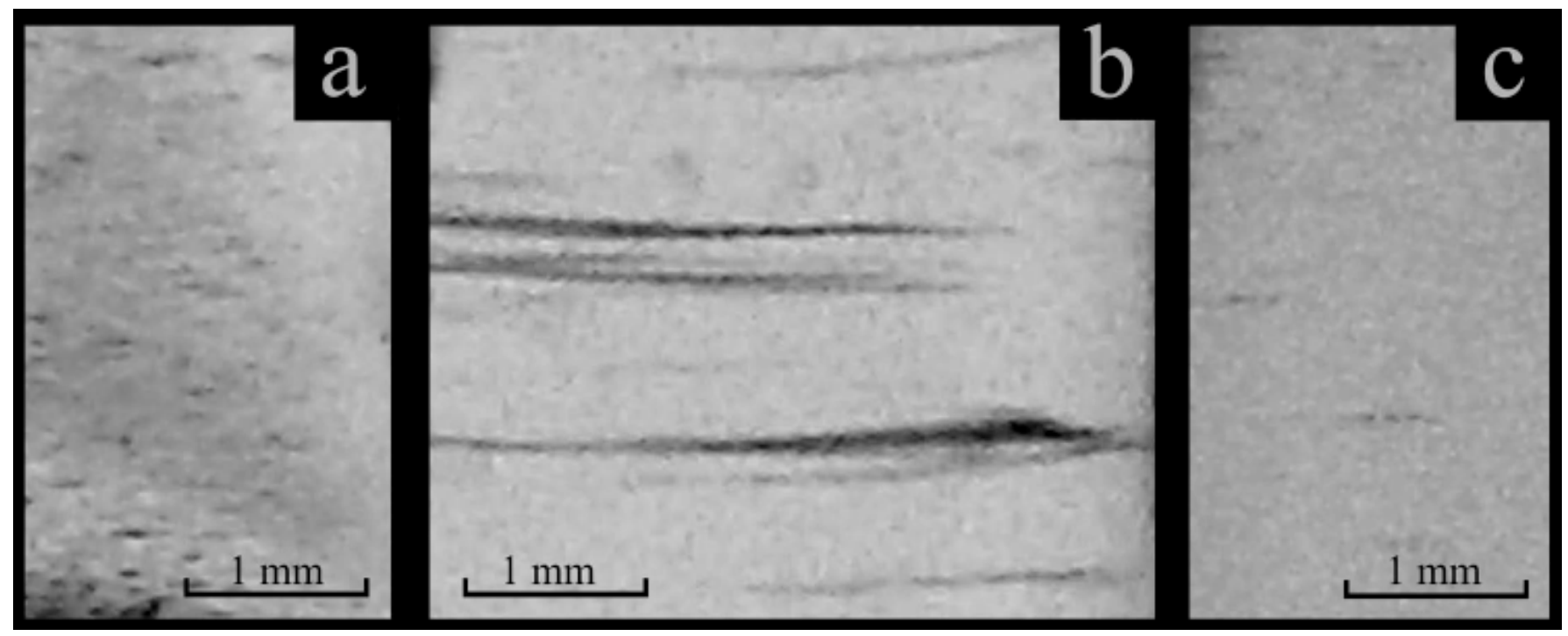

Fig. (4). The photos of linear aggregates formed in three different systems under the influence of external magnetic field: (a) - nanoparticles dispersion; (b) - FG (3 mg/ml) solution containing nanoparticles; (c) - BSA (10 mg/ml) solution containing nanoparticles. 
with same characteristics. The advantage of spin label technique is that it allows obtaining information not only about macromolecule adsorption but also about local viscosity which defines rotational mobility of the label. Globular protein BSA is characterized by the highest rotational correlation time $\tau$ in solution. Evidently, the reason of this is close packing of BSA coil. This protein adsorb in smaller amount on magnetite nanoparticles than PEI or FG.

Micron-size linear aggregates form under the influence of direct magnetic field. These aggregates consist of nanoparticles and adsorbed macromolecules. Analysis of aggregation kinetics dependence on nanoparticles and protein concentration in solution and external magnetic field strength is the object of follow-up study.

We should note that spin label technique can be used also in the study of macromolecule adsorption on non-magnetic nanoparticles. In this case the main characteristic parameter is rotational correlation time of labels. This parameter changes sharply when macromolecules immobilize by adsorbent surface [4, 5].

\section{ACKNOWLEDGEMENTS}

The authors gratefully acknowledge V.I. Filippov for providing of magnetite hydrosol, V.B. Leonova for the help in sample preparation, prof. M.A. Rosenfeld for providing of proteins, light scattering data and useful discussion of results obtained.

The work was supported by the Russian Foundation for Fundamental Research (Project No. 08-04-00632).

\section{REFERENCES}

[1] Fox, K.K.; Robb, I.D.; Smith, R. Electron paramagnetic resonance study of the conformation of macromolecules adsorbed at the solid/liquid interface. J. Chem. Soc. Faraday Trans., Part I, 1974, 70, 1186-90.

[2] Hommel, H.; Legrand, A.P.; Balard, H.; Papirer, E. Influence of chain length on conformations of poly(ethylene glycol) chains grafted on silica. Polymer, 1983, 24 (8), 959-63.

[3] Miller, W.G.; Rudolph, W.T.; Veksli, Z.; Coon, D.L.; Wu, C.C.; Liang, T.M. Spin label studies of polymer motion at or near an interface. In Molecular Motion in Polymers by ESR; Boyer, R.E.; Keinath, S.E., Ed.; MMI press: Chur, 1980; pp. 145-165.

[4] Kovarski, A.L.; Yushkina, T.V.; Tikhonov, A.P. Surfaces spin labeling - New approach to studying macromolecules interaction with the solid surface and properties of boarder layers in polymer composites. In New perspectives in Chemistry and Biochemistry; Zaikov, G.E., Ed.; Nova Science Publishers, Inc.: New York, 2002; pp. 1-3.
[5] Kovarski, A.L.; Yushkina, T.V.; Kasparov, V.V.; Tikhonov, A.P. Surface spin labeling in interface layers of filled polymers investigation. Colloid Polym. Sci., 2003, 281 (4), 308-18.

[6] Sorokina, O.N.; Bychkova, A.V.; Kovarski, A.L. Analysis of FMR spectra of magnetite nanoparticles aggregates formed by magnetic field. Zhurnal Khimicheskaya Fizika (in Russian), 2009, 28 (4), 927.

[7] White, A.; Handler, P.; Smith, E.L.; Hill, R.L.; Lehman, I.R. Principles of biochemistry, 6th ed.; McGraw-Hill, Inc: New York, 1978.

[8] Freed, J.H. Theory of slow tumbling ESR spectra for nitroxides. In Spin Labeling: theory and applications; Berliner, L.J., Ed. Academic press: New York, 1976; pp. 53-132.

[9] Shapiro, A.B.; Bogach, L.S.; Suskina, V.I., Chumakov, V.M., Rozantsev, E.G. Russ. Chem. Bull., 1975, 9, 2077-81.

[10] Kovarski, A.L.; Sorokina, O.N. Study of local magnetic fields and magnetic ordering in fluid and solid matrices containing magnetite nanoparticles using TEMPOL stable radical. J Magnet Magn Mate., 2007, 311, 155-61.

[11] Antsiferova, L.I.; Vasserman, A.M.; Ivanova, A.N.; Livshits, V.A.; Nazemets, N.S. Electron Spin Resonance Spectra of Spin Labels and Probes; Nauka; Moscow, 1977.

[12] Abragam, A. Principles of Nuclear Magnetism; Oxford University Press: New York, 1961.

[13] Noginova, N.; Weaver, T.; King, T.; Bourlinos, A.V., Giannelis, E.P., Atsarkin, V.A. NMR and spin relaxation in systems with magnetic nanoparticles. J. Phys. Cond. Matter, 2007, 19, ID $076210(10 \mathrm{pp})$.

[14] Sorokina, O.N.; Kovarski, A.L.; Bychkova, A.V. Application of paramagnetic sensors technique for the investigation of the systems containing magnetic particles. In Progress in Nanoparticles research; Frisiras, C.T., Ed.; Nova Science Publishers: New York, 2008; pp. 91-102.

[15] Bachmann, L.; Schmitt-Fumain, W.W.; Hammel, R.; Lederer, K. Size and shape of fibrinogen, 1. Electron microscopy of the hydrated molecule. Makromol. Chem., 2003, 176 (9), 2603-19.

[16] Bondi, A. Physical Properties of Molecular Crystals; Wiley: New York, 1968.

[17] Kovarski, A.L. New Concepts in Polymer Science. Molecular Dynamics of Additives in Polymers. VSP: Utrecht, 1997, p.64.

[18] Vasserman, A.M.; Kovarskii, A.L.; Yasina, L.L.; Buchachenko, A.L. Exchange and dipole interactions and local concentrations of stable radicals in polymers. Theor. Exp. Chem., 1977, 13 (1), 1923.

[19] Anisimov, O.A.; Nikitaev, A.T.; Zamaraev, K.I.; Molin, Yu.N Separation of exchange and dipole-dipole broadening on the basis op viscosity changes in ESR spectra. Theor. Exp. Chem., 1974, 7 (5), 556-9.

[20] Taketomi, S.; Chikazumi, S. Magnetic fluids-Principle and Application; Nikkan Kogyo Shinbunsha: Tokyo, 1988 [in Japanese], Russian Version, Mir Publisher, Moscow, 1994.

[21] Sorokina, O.N.; Kovarski, A.L. Peculiarities of electron magnetic resonance spectra of the linear aggregates of ferromagnetic nanoparticles. In Chemical and Biochemical Physics, Kinetic and Thermodynamics; Stott, P.E.; Zaikov, G.E.; Kablov, V.F., Eds.; Nova Science Publishers: New York, 2007; pp. 49-55. 